\title{
Maurice Pardé, potamologue (1893-1973)
}

\author{
Jean de Beauregard
}

Grenoble a célébré dignement son centenaire le jour de sa fête, le 22 septembre 1993.

La cérémonie réunit autour de sa famille un grand nombre d'amis et d'anciens élèves, certains devenus ses collègues. Elle se tint à l'Institut de Géographie Alpine où il enseigna et qui conserve dans le Fonds Maurice PARDÉ la documentation hydrologique exceptionnelle qu'il avait rédigée et rassemblée.

Mme Huguette Vivian, Directeur de Recherche au CNRS, est à l'origine de cette cérémonie et du Fonds. Elle mérite la reconnaissance du monde scientifique.

Comme prévu lors de cette cérémonie, La Houille Blanche rappelle ici la vie, la personnalité et l'œuvre de Maurice PARDÉ.

\section{Une trajectoire brillante et insolite}

De Senlis à Nancy, son itinéraire de près de 80 ans figure dans le tableau 1 avec ses principales étapes. Grenoble y occupe la première place, celle de ses doctorats, de son enseignement, de sa retraite, donc de la quasi-totalité de sa carrière longue d'un demi-siècle. Car jusqu'à la fin de sa vie, malgré la maladie, il donna conseils et avis de vive voix ou par lettres. Il fut membre du jury de thèse de Melle Monique DACHARRY, à Brest, deux mois avant de disparaître.

Sa valeur fut reconnue à l'étranger. Belgrade, Berlin, Genève, Rome et Vienne lui conférèrent la qualité de membre d'honneur de leur Société de Géographie. L'Université de Bonn le fit Docteur honoris causa en 1963. Et la France lui ouvrit les portes de l'Institut en lui donnant le titre de Correspondant de l'Académie des Sciences en 1965. Le président du Comité technique de la Société Hydrotechnique de France, M. E.-G. BARILlon, lui-même académicien, ne fut pas étranger à cet honneur.

Cursus original, voire paradoxal.

Il est déjà rare d'être, selon son expression, «bidocteur ", surtout en Lettres et Sciences.

Il est plus rare encore d'exercer dans les 2 Facultés en même temps. La bonne entente entre Raoul BLANCHARD et le Doyen des Sciences René Gosse permit cette audace à la mesure de l'intéressé.

Enfin, qu'un géographe de formation littéraire accède à l'Institut par l'Académie des Sciences prouve non seulement l'estime portée à l'élu mais aussi l'objectivité et la largeur d'esprit de ceux qui l'accueillirent.

Maurice PARDÉ fait éclater les cadres habituels sous l'effet de sa puissante personnalité.
Tableau 1. Etapes de la vie de Maurice Pardé

\begin{tabular}{|c|c|}
\hline décembre 1893 & Naissance à Senlis (Oise) \\
\hline 913-1915 & $\begin{array}{l}\text { Etudiant à Nancy et Dijon. Licence ès } \\
\text { Lettres. }\end{array}$ \\
\hline $1915-1921$ & $\begin{array}{l}\text { Professeur délégué au Puy, à Grenoble, } \\
\text { à Lyon. }\end{array}$ \\
\hline 919 & $\begin{array}{l}\text { Premières publications hydrologiques: } \\
\text { "Les crues des rivières cévenoles " } \\
\text { "Le régime du Rhône à Beaucaire" }\end{array}$ \\
\hline vril 1925 & Doctorat ès Lettres à Grenoble \\
\hline 1923-1929 & Professeur à Bourg-en-Bresse \\
\hline 1929-1947-1966 & $\begin{array}{l}\text { Professeur chargé de cours à l'Ecole des } \\
\text { Ingénieurs Hydrauliciens (Institut 'Poly- } \\
\text { technique) } \\
\text { Professeur de potamologie à la Faculté } \\
\text { des Sciences de Grenoble }\end{array}$ \\
\hline $1932-1935-1966$ & $\begin{array}{l}\text { Maître de Conférences à la Faculté des } \\
\text { Lettres } \\
\text { Professeur de géographie physique et de } \\
\text { potamologie à la Faculté des Lettres de } \\
\text { Grenoble }\end{array}$ \\
\hline 1947 & Doctorat ès Sciences à Grenoble \\
\hline 1958 & $\begin{array}{l}\text { Président de la Commission d'Hydrologie } \\
\text { fluviale du Comité National français de } \\
\text { Géographie }\end{array}$ \\
\hline 1965 & $\begin{array}{l}\text { Correspondant de l'Académie des Scien- } \\
\text { ces }\end{array}$ \\
\hline $1^{\text {er }}$ oc & Professeur honoraire \\
\hline 14 juin 1973 & Décès à Nancy \\
\hline
\end{tabular}

\section{Un tempérament créateur}

Le berceau de sa famille est le Gâtinais, autour de Montargis, non loin d'Orléans, patrie de son maître Raoul BLANCHARD. Pour ces enfants du plat pays (de plus, la thèse de R. BLANCHARD porte sur la Flandre) il fallait vivre, semble$\mathrm{t}$-il, près des cimes et des torrents fougueux.

Fils et père de forestiers, Maurice PARDÉ a baigné dès son jeune âge dans le monde de la nature. Il en a découvert la beauté, le rythme et la complication des relations entre ses composants. Avec son père il a publié, en 1938, dans la collection Armand Colin, le livre «Arbres et Forêts ».

La coxalgie qui le frappe à 12 ans lui interdit le métier des armes dont il rêvait et lui laisse une légère claudication. Pendant deux ans, éloigné de toute scolarité régulière, il entreprit de dévorer des ouvrages de littérature, de l'Iliade à nos grands classiques, accompagnés des commentaires de BRUNETIÈRE et LANSON, tout en poursuivant ses lectures historiques avec THIERS, MASPERO et FERRERO. L'histoire militaire le fascinait avec une prédilection pour la guerre de Sécession.

L'épreuve le mûrit prématurément. Il présente déjà une combinaison rare chez le même homme de qualités extrêmes, portées presque jusqu'au paroxysme. 
Une vitalité débordante: il s'instruit «avidement, et même avec une vraie fièvre ", une " curiosité dévorante". Mais il éprouve aussi le souci de servir par un «dévouement sans réserve à une grande cause ", au besoin par le journalisme et la politique.

Un homme de caur, simple, chaleureux avec ses étudiants témoins de son dévouement, avec les ingénieurs, hydrauliciens, statisticiens, cheminots, quémandeurs de ses conseils gratuits, avec ses secrétaires, «les bouelles» bénéficiaires de petits cadeaux, et jusqu'à son marchand de disques qui tenait sans doute en lui son meilleur client.

Une mémoire d'ordinateur, qui conserve tous les chiffres de débits moyens, de crues, d'étiages des fleuves et rivières à leurs stations de mesure, mais aussi les détails des grandes campagnes militaires en Europe et en Amérique, qui se souvient de la crue du Danube fort préjudiciable à la retraite d'Allemagne en octobre 1813 et du nom des régiments et de leur colonel engagés dans la bataille de la Marne en septembre 1914, qui connaît les œuvres musicales, leurs divers mouvements, les instruments qui introduisent les thèmes et les interprètes, enfin, un indicateur de chemins de fer qui, à la demande d'un collègue géologue, en 1937, lui donne les horaires des trains successifs, avec les gares frontières, d'un voyage de quatre jours entre Grenoble et Moscou.

Une soif $d$ 'indépendance, qui soulève un paradoxe réconfortant pour la curiosité scientifique pure. La démarche de M. PARDÉ est commandée par ses goûts : faire ce qui lui plait, personnellement, presque égoïstement. Il supporte mal «tout travail imposé par d'autres. En se rappelant cette époque de sa vie (18 ans), il n'a d'ailleurs jamais prétendu la donner en modèle à suivre ". " Il ne manquait ni d'ardeur ni de volonté pour se vouer aux entreprises qui plaisaient à ses caprices impérieux. " Or, ses travaux ont une valeur appliquée inestimable, non seulement pour les hydrauliciens, hydroélectriciens, ingénieurs des eaux et forêts, mais aussi urbanistes, édiles, habitants de régions victimes d'inondations comme en 1992, 1993 et 1994 à Vaison-la-Romaine, Bédarrides, Saint-Jean-de-Maurienne, Valréas, Bollène et le BasRhône. Si les responsables de la région méridionale tenaient compte de l'observation de M. PARDÉ que les crues les plus dévastatrices y sévissent surtout entre le 10 septembre et le 22 octobre (Corse exclue) et déclaraient cette période "à risques ", la prévention se développerait plus efficacement.

Un esprit rigoureux et scrupuleux. Si l'hydrométrie remonte aux Pharaons avec le Nilomètre d'Assouan, l'hydrologie scientifique, esquissée par Pierre PERrault en 1674 avec « De l'origine des fontaines », date des travaux de M. PARDÉ, 250 ans plus tard. Il a, le premier, recensé, analysé et relié causes et effets de l'écoulement, tracé les frontières de cette discipline et défini son objet : la mesure physique du débit, débit liquide, débit solide, et ses variations en fonction des facteurs, climat (précipitations, température), relief, sol, végétation, et de plus en plus l'homme lui-même, variations qui constituent le régime.

Qu'aujourd'hui et demain on multiplie les mesures physiques (température), chimiques $(\mathrm{pH})$, biologiques et bactériologiques élargit le champ d'étude et dilate l'hydrologie, science des rivières, au-delà de la notion du bilan : pluie-débit.

M. PARDÉ préconisa d'ailleurs, dans les années 20 , la création d'un centre d'études des eaux continentales. Il aurait suscité des séries de mesures propices à un traite- ment statistique bien étayé. Un texte de lui sur les modules ou sur les crues comporte toujours l'examen des facteurs, du phénomène, des effets. C'est logique et complet, mais nouveau et insolite, encore trop souvent aujourd'hui. Sur les variations des débits ou régimes, il a poussé l'analyse jusqu'au bout en distinguant les régimes simples (ex. nival de montagne), complexes originels (ex.: nivopluvial), complexes changeants (Rhin, Rhône), notions souvent nouvelles.

40 pages sur le « Rapport entre l'intensité des pluies et les débits maxima des crues » dans la Revue pour l'Etude des Calamités, Genève, 1939, demeurent un modèle inégalé, et trop méconnu. Un quart de considérations théoriques sur le mécanisme du ruissellement, sa décoordination selon l'étendue de la surface réceptrice, 3/4 d'exemples.

Avec un esprit exigeant et souple, son souci d'épouser la réalité dans sa complexité le conduit à confesser ses difficultés et ses erreurs. " J'ai dit des énormités dans ma thèse " l'ai-je entendu dire, médusé, dans l'un de ses premiers cours de l'année. Sa thèse qui «fut sa légende vécue, son épopée». En 1942, il publia «Quelques nouveautés sur le Régime du Rhône " (Erratum et Addendum à un gros ouvrage) en 200 pages. Dans l'introduction à son ouvrage inédit, sur « l'Abondance moyenne annuelle des rivières ", il intitule certains paragraphes : «Insuffisance et erreurs certaines de ce livre ", "Insuffisance relative de notre effort personnel ». Rien d'un pontife!

De multiples " marottes " ou pôles d'intérêt. Outre l'histoire et les chemins de fer déjà évoqués, les sports le passionnaient. Je l'ai rencontré un dimanche au stade de Grenoble lors d'une rencontre de football (de seconde division). Le rugby avait sa préférence pour l'engagement physique et la souplesse qu'il requiert. Lors de ses séjours à Paris, il ne manquait pas une visite au Louvre ou à une exposition de peinture, de Goya entre autres dont les délires sur le diable l'éblouissaient. Enfin, il faut revenir sur la musique qui l'accompagna toute sa vie. WAGNER y tenait la première place à cause de ses thèmes héroïques ou éternels : l'amour, l'honneur, la mort, suivi de tous les compositeurs classiques, car il savait être éclectique. Il aimait faire partager ses émotions au cours de «concerts " improvisés.

Tant de qualités humaines réunies dans cette force de la nature, en particulier sa mémoire, lui assurèrent une puissance de travail stupéfiante, comme la rédaction en un jour de 40 pages truffées de chiffres. Elles expliquent l'ampleur de son œuvre potamologique.

\section{Le fonds Maurice Pardé}

\section{(Institut de Géographie Alpine 17, rue Maurice Gignoux 38031 Grenoble Cedex)}

La potamologie est un mot qu'il a créé pour désigner « la science des fleuves et des rivières ".

Sa production écrite, rassemblée dans le Fonds Maurice PARDÉ, se répartit sous trois rubriques:

1 - l'œuvre imprimée,

2 - des pages inédites, comme ses souvenirs sur Raoul Blanchard, et surtout sa correspondance,

3 - deux ouvrages achevés, en " tapuscrits ", qui attendent et méritent une publication. 


\subsection{L'auvre imprimée}

Sa contribution hydrologique tient en 300 titres, dont 120 relatifs aux crues, soit $40 \%$. Elle est donc marquée par l'abondance et un penchant pour les paroxysmes.

On peut distinguer les monographies de bassins et les sujets généraux, où se rangent ses huit œuvres majeures :

\section{Monographies}

1. "Le régime du Rhône»

( 1 bis. "Quelques nouveautés sur le régime du Rhône "

2. "Le régime du Mississipi »

3. «Le régime de la Garonne»

4. "Les régimes fluviaux de la péninsule ibérique »

5. «Etudes potamologiques sur la Loire et ses affluents ». Bref, surtout les fleuves français, sauf la Seine, de tempérament placide

\section{Thèmes généraux}

6. "Fleuves et Rivières "

7. "Questions diverses relatives à l'Hydrologie fluviale »

8. "Sur la puissance des crues en diverses parties du monde "

«Fleuves et Rivières » comprend trois parties: les facteurs des régimes, mesures et calculs hydrométriques, les éléments des régimes. Ses rééditions confirment sa valeur de référence.

Du Rhône, «le plus complexe de tous les fleuves ", à «Fleuves et Rivières ", l'ouvrage de base, et à l'inventaire classé par types des grandes crues mondiales, le parcours est exemplaire, mais, nous allons le voir, inachevé.

\subsection{Pages inédites - correspondance}

Généreux et même prodigue, Maurice PARDÉ écrivait beaucoup. Il a couvert des cahiers et des carnets de notes qui montrent le soin scrupuleux apporté à ses lectures, à leurs analyses, qu'elles alimentent ensuite un compte rendu ou simplement qu'elles apparaissent dans la bibliographie de ses livres.

Sur son maître Raoul BLANCHARD (1877-1965) il a rédigé un long texte de souvenirs qui mériterait une publication. Le récit de la rencontre de deux personnalités aussi différentes mais également puissantes est une page d'anthologie. Il souligne son invention en géographie urbaine des notions nouvelles de situation générale et de site local d'une agglomération. Ainsi, Grenoble, ville de confluence de rivières et de contacts de régions naturelles, est aussi la ville du Pont, seul ouvrage entre Albertville et Saint Gervais qui assure en permanence le passage audessus d'un cours d'eau capricieux, parce que les alluvions du Drac plaquent l'Isère contre la Chartreuse et l'empêchent, là, de divaguer.

Maurice PARDÉ a entretenu une correspondance débordante, qui remplirait des livres, avec le monde entier scientifique et technique, quêtant des informations, commentant les résultats des jaugeages. Il y a là un gisement à exploiter. Comment ? Que les destinataires qui ont conservé ses envois les retournent à Grenoble, au Fonds Maurice PARDÉ, dans l'intérêt de tous les hydrologues.

\subsection{Ouvrages à publier}

Toujours selon le même souci de ne rien perdre du savoir de ce grand potamologue et de le diffuser dans le public spécialisé, il faut souhaiter que nous parvienne son message posthume : plus de 1500 pages dactylographiées
Thèse de Lettres, Erratum et Addendum à un gros ouvrage Rev. de Géo. Alpine

R.G. des Pyrénées et du SO

Rev. de Géo. de Lyon

Norois

1963 (1961) Geog. Saragosse
(« tapuscrits »), datées de 1970 environ, corrigées, prêtes à l'impression (sous contrôle). Elles forment la matière de 2 ouvrages :

- une nouvelle rédaction de la Potamologie, prévue pour la collection «Orbis », en 2 tomes :

- L'Hydrologie fluviale, 375 pages ;

- La Dynamique fluviale, plus de 500 pages ;

- l'Abondance moyenne annuelle des rivières, 660 pages.

La Potamologie suit un plan conforme aux traités antérieurs de l'auteur. Les questions sont élargies et approfondies, avec davantage de développements mathématiques et la prise en compte des publications récentes (en 1970) françaises et étrangères. La bibliographie est très développée, bien que qualifiée de "sommaire".

L'Hydrologie fluviale contient 8 chapitres énumérés tableau 2. Le chapitre VII, les crues, apparaît en détail dans le tableau 3. La Dynamique fluviale contient 20 chapitres énumérés tableau 4.

L'Abondance, fraction du $1^{\mathrm{er}}$ tome, l'Hydrologie fluvia$l e$, est devenue un gros livre, riche d'informations chiffrées et de réflexions critiques : une référence incomparable. Les 23 chapitres apparaissent dans les tableaux $5 \& 6$, après les sous-titres de l'Introduction qui rendent bien le style et la manière de l'auteur.

Pour être plus explicite et mettre le lecteur en appétit, le tableau 7 complète la présentation de l'Abondance.

Les raisons de publier, c'est-à-dire de mettre à la disposition du public, méritent d'être sérieusement prises en considération.

1. La lecture des textes et l'élaboration des 20 pages de tables des matières de ces 2 ouvrages montrent qu'ils sont achevés, prêts à l'édition.

2. La quantité d'informations chiffrées. Reliques précieuses.

3. La qualité reconnue et permanente, car la valeur de l'analyse naturaliste demeure.

4. Les séries de mesures "naturelles », qui permettent d'appréhender les régimes « naturels » ou géophysiques, se raréfient. Les influences artificielles (barrages, endigue- 
ment, prélèvements) augmentent. Le Colorado devient « A River No More " (Fradkin).

5. Les moyens nouveaux de reproduction informatique ou électronique disques, CD-Rom, etc...

6. Les organismes intéressés ne manquent pas : Université, Agences de l'Eau, Cemagref, DE, CNR, EDF, ...
Les dégâts récents coûtent beaucoup plus cher et seraient réduits si l'on connaissait mieux l'hydrologie, la potamologie pardéenne.

Ce serait comme une contribution à l'Institut de l'Eau projeté par M. PARDÉ, il y a 75 ans, et le couronnement de son centenaire.

\section{Tableau 2. Hydrologie fluviale -8 chapitres et paragraphes}

\section{I - LES FACTEURS DE L'HYDROLOGIE FLUVIALE}
A. Le relief
B. Le climat
C. La nature du sol
D. La végétation
E. Travaux humains

\section{II - LES BASES HYDROMÉTRIQUES ET THÉORIQUES}

\section{DE LA POTAMOLOGIE}

A. Détermination des hauteurs

B. Détermination directe des débits

C. Détermination des débits par des formules hydrologiques ou géographiques

D. Détermination des débits par des procédés essentiellement mathématiques

E. Etudes hydrologiques approfondies sans hautes mathématiques

\section{III - L'ABONDANCE MOYENNE ANNUELLE}

A. Le module et le bilan Précipitations-Débits

B. Les facteurs et quelques valeurs du déficit annuel d'écoulement

C. Les modules spécifiques

D. Les modules bruts

E. Variation des modules dans l'espace

F. Variation de l'abondance dans le temps

\section{IV - LES VARIATIONS SAISONNIĖRES}
A. Importance
B. Moyens d'expression
C. Les régimes simples
D. Régimes complexes originels
E. Régimes complexes par combinaisons

\section{$\mathrm{V}$ - LES FRÉQUENCES}

A. Intérêt des études relatives aux fréquences

B. Les fréquences partielles

C. Fréquences cumulées. Courbes des débits classées

$\mathrm{VI}$ - LES ÉTIAGES

A. Saisons, définitions et expressions numériques

B. Causes des étiages

C. Courbes de décroissance

D. Etiages énormes

E. Etiages par catégories fluviales saisonnières avec chiffres types caractéristiques

F. Catégories fluviales sans chiffres types pour les étiages spécifiques

G. Quelques valeurs brutes pour les étiages records

VII - LES CRUES
A. Définitions
B. Causes
C. Allure et genèse des crues dans l'espace et dans le temps
D. Rapports entre les crues et les apports d'eau atmosphérique
E. Puissance des crues. Généralités
F. Débits maxima dans le monde. Bassins petits et moyens
G. Débits maxima dans les grands et très grands bassins
$\mathrm{H}$. L'homme et les crues

VIII - L'ÉROSION ET LES TRANSPORTS SOLIDES
A. La force tractrice des courants
B. Transports de fond
C. Transports en suspension
D. Transports en dissolution
E. Tonnages combinés de toutes espèces
$\mathrm{F}$. Les volumes des érosions et des remblaiements

\section{BIBLIOGRAPHIE}

Tableau 3. Hydrologie fluviale - ch. VII - les crues

\section{A. DÉFINITIONS \\ B. CAUSES}

1. Les embâcles

2. Les débâcles

3. La fonte des neiges

a) Bassins petits et moyens

b) Grands et très grands bassins

c) Crues d'origine à la fois nivale et pluviale

4. Les pluies génératrices des crues

a) Définitions numériques des pluies

b) Notions anticipées sur les resultats fluviaux possibles, en théorie, des intensités pluviales

c) Pluies maxima locales

d) Pluies moyennes sur diverses surfaces réceptrices

5. Situations atmosphériques responsables

a) Nécessité de perturbations atmosphériques

b) Situations atmosphériques et averses typiques ou aberrantes

\section{ALLURE ET GENĖSE DES CRUES DANS L'ESPACE ET DANS LE TEMPS}

1. Vitesses de translation

2. Les évolutions. Monogénie et polygénie

a) Evolutions influencées par des troubles dans les translations

b) Evolutions d'origine simple ou monogénique

c) Polygénie sans concordances entre flots élémentaires sur le collecteur principal

d) Polygénie avec concordances entre flots élémentaires différents décalés dans le temps à leur origine 


\section{RAPPORTS ENTRE LES CRUES ET LES APPORTS D'EAU ATMOSPHÉRIQUE}

1. Rapports entre les débits maxima fluviaux et les pluies a) Mécanismes

b) Quelques valeurs types remarquables des rapports

2. Les quotients ou coefficients d'écoulement des crues

a) Rôle de l'intensité pluviale

b) Influences des températures et des saisons

c) Influence très déterminante de la saturation préalable

d) Influence de la nature du sol

e) Rôle des neiges

3. Les déficits d'écoulement de crue

a) Considérations générales

b) Quelques ordres divers de grandeurs

c) Influences variées de la saturation préalable forte ou faible

d) Pertes très accrues dans les terrains extrêmement absorbants

E. PUISSANCE DES CRUES. GÉNÉRALITÉS

17 pages

1. Volumes totaux

a) Délimitation des volumes à considérer

b) Débits de base et calcul des volumes totaux nets

c) Quelques chiffres de volumes totaux

2. Débits maxima

a) Leur appréciation numérique

F. DÉBITS MAXIMA DANS LE MONDE. BASSINS PETITS ET MOYENS

1. Chiffres exorbitants

a) Valeurs-types

b) Texas

c) Japon

d) Autres régions

2. Chiffres formidables

a) Crues méditerranéennes

b) Nouvelle-Zélande et Australie

c) Régions tropicales diverses

d) Asie des Moussons et notamment Inde

e) Etats-Unis

3. Crues très fortes

a) Valeurs-types

b) Etats-Unis

4. Crues fortes ou assez fortes

5. Crues modérées, faibles, insignifiantes

G. DÉBITS MAXIMA DANS LES GRANDS ET TRĖS GRANDS BASSINS

1. Facteurs combinés des coefficients et principes de l'examen

a) Instabilité au moins relative des coefficients de l'amont à l'aval

b) Cas de décroissances générales pour $A$ et $A^{\prime}$ vers l'embouchure

c) Cas d'augmentation générale pour $A$ et $A^{\prime}$ vers l'embouchure

d) Rôle capital de la polygénie dans les grands bassins

e) Coefficients records mondiaux bien moindres pour les grands bassins que pour les surfaces réceptrices petites et moyennes

2. Fleuves ayant des crues très faibles à leurs embouchures

3. Crues médiocres vers la fin des cours

4. Crues modérées ou assez fortes vers la fin des cours

5 . Fortes crues sur les cours inférieurs après des vicissitudes diverses
a) Rhin et Rhône
b) Fleuves ibériques
c) Pô
d) Mississipi

6. Crues les plus fortes du monde sur les grands et très grands bassins a) Etats-Unis

b) Brésil méridional et Uruguay

c) Asie des Moussons

7. Fleuves à bassins immenses

a) Fleuves sibériens

b) Congo et Amazone

\section{H. L'HOMME ET LES CRUES}

1. Hauteurs maxima sur les zéros ou sur les étiages

2. Etendues inondées et profondeurs de submersion

3. Dommages causés par les grandes crues

4. Prévisions des crues déjà déclenchées

5. Prévision des pluies ou des fontes dangereuses

6 . Etudes sur la grandeur des crues à escompter

7. Prévisions sur les fréquences saisonnières

8. Remèdes
a) Reboisement
b) Endiguements longitudinaux
c) Aménagement des lits
d) Les barrages-réservoirs 
J. DE BEAUREGARD

Tableau 4. Dynamique fluviale -20 chapitres

INTRODUCTION

PREMIĖRE PARTIE - LES COURANTS FLUVIAUX

I - Les causes et les caractéristiques générales des vitesses fluviales

II - Les jaugeages

III - Les formules de I'hydraulique

IV - Les chiffres des vitesses dans les cours d'eau

$\mathrm{V}$ - La turbulence

DEUXIĖME PARTIE — LES DÉBITS SOLIDES DES COURS D'EAU

VI - Les forces tractrices des courants fluviaux. Appendice

VII - Transports de fond ou de matériaux grossiers

VIII - Les transports en suspension

IX - Transports en dissolution

$X \quad$ - Transports totaux de toutes espèces

$\mathrm{XI}$ - Volumes des érosions et des remblaiements

TROISIĖME PARTIE - LES CARACTÉRISTIQUES ET LES AMÉNAGEMENTS NATURELS DES LITS

XII - Les lits fluviaux. Leurs différentes espèces

III - Interconnexions des formes fluviales caractéristiques, et aménagements à courants libres

XIV - Déformations verticales rapides

XV - Déformations verticales durables

XVI - Les profils en long actuels

XVII - Déformations latérales et méandres

XVIII - Divagations des lits

$\mathrm{XIX}$ - Les profils en travers

$X X$ - Les matériaux des lits

CONCLUSIONS À LA FIN DE CHAQUE PARTIE

BIBLIOGRAPHIE À LA FIN DE CHAQUE CHAPITRE

BIBLIOGRAPHIE GÉNÉRALE À LA FIN DE L'OUVRAGE

Tableau 5. L'Abondance - 23 chapitres

INTRODUCTION - ORIGINE ET OBJETS DE CET OUVRAGE

1 - L'abondance moyenne, élément considérable de la Potamologie

2 - Les nodules et les bilans annuels de l'écoulement. Définitions et intérêts

3 - Plaidoyer pour l'ampleur insolite de notre texte

$4 \quad$ - Insuffisance et erreurs certaines de ce livre

5 - Insuffisance relative de notre effort personnel

6 - Notre excuse partielle par la limitation de nos forces

$7 \quad$ - Utilité possible ou même probable de ce livre

8 - Plaidoyer pour l'insuffisance de notre illustration cartographique

9 - Matières traitées en chaque partie de notre livre

PRÉAMBULE TECHNIQUE — LA DÉTERMINATION DES DÉBITS FLUVIAUX

PREMIÈRE PARTIE - LES BILANS ANNUELS PRÉCIPITATIONS DÉBITS

I - Définitions et quelques faits primordiaux

II - Les facteurs du déficit annuel d'écoulement

III - Les facteurs du déficit annuel (suite)

IV Z Les valeurs des déficits globaux d'écoulement dans l'espace

VI - Les valeurs des quotients annuels d'écoulement

DEUXIÈME PARTIE - LES VALEURS DE L'ABONDANCE GLOBALE DANS LE MONDE

VII - Les modules dans les diverses régions terrestres

VIII - Variations de l'abondance annuelle de l'amont à l'aval

IX - Volumes liquides totaux apportés aux océans par les fleuves

TROISIĖME PARTIE - MODIFICATIONS DES MODULES DANS LE TEMPS

$X$ - Données statistiques à établir et à interpréter

$X I \quad$ - Les types effectifs de distributions pour les modules particuliers

XII - Rapports des modules extrèmes

XIII - Ecarts moyens des modules particuliers avec les modules globaux

XIV - Ecarts des modules globaux partiels avec la normale

$X V$ - Renforcements ou diminutions très durables dans les mêmes sens respectifs de l'abondance fluviale

$X \mathrm{VI}$ - Prédictions sur l'abondande moyenne

QUATRIÈME PARTIE — FORMULES ET MÉTHODES POUR L'ÉVALUATION DES MODULES

XVII - Formules d'évaporation

XVIII - Formules pour les modules globaux

$\mathrm{XIX}$ - Extensions à la normale

XX - Les modules particuliers calculés d'après les précipitations

$X X I$ - Les modules particuliers. L'année dite hydrologique

$X X I I$ - Les modules particuliers. Retouches diverses et ajustements pour l'emploi des corrélations

XXIII - Evaluations relatives aux modules particuliers des cours d'eau glaciaires

APPENDICE — INDICES D'ARIDITÉ ET D'HIMIDITÉ

CONCLUSIONS D'ENSEMBLE - AMPLEUR INSOLITE DE CE TEXTE CONCLUSIF

A - Evaluation des modules particuliers

B - Evaluations des modules globaux normaux

C - Variabilité des modules

D - Les expressions numériques des bilans précipitations-débits dans le monde

E - Les modules globaux

REMARQUES FINALES 
INTRODUCTION - Origine et objet de cet ouvrage

1 - L'abondance moyenne, élément considérable de la Potamologie

Nous présentons ci-dessous, dans ce livre, une étude relative à l'Abondance moyenne des rivières. Selon nos intentions primitives, ce texte devait comprendre seulement une ou deux centaines de pages à inclure dans un manuel de Potamologie générale. Plus précisément, ces chapitres devaient figurer, entre autres matières, dans le Premier Tome : Hydrologie fluviale, du traité en question, le deuxième tome étant consacré à la Dynamique fluviale.

Nous devons aussitôt définir ces mots qui correspondent essentiellement à notre terminologie personnelle.

La Potamologie est la science des eaux courantes intérieures, et surtout des eaux fluviales et des lits fluviaux. Par simplification, nous appelons fréquemment : rivières, non seulement les cours d'eau que l'on désigne d'habitude par ce mot, mais encore les fleuves, ruisseaux, torrents, etc... Et, dans un même souci, faute d'adjectif tel que " riviéral » (1), nous qualifions de fluviaux tous les phénomènes qui se passent dans ces organismes petits ou grands.

Puis, I'Hydrologie fluviale, première subdivision de la Potamologie, décrit et explique les régimes fluviaux, c'est-à-dire les caractères et les variations en débits des eaux courantes. La Dynamique fluviale a pour objet les courants fluviaux, les transports par eux de matières solides, puis les lits fluviaux, les actions des courants sur ces derniers et les influences réciproques.

Bien entendu les explications relatives à la Potamologie doivent invoquer largement et donc bien connaître tous les facteurs géographiques naturels, climat, relief, nature du sol, végétation, et de plus en plus maintenant, les causes artificielles, c'est-à-dire introduites par l'homme : endiguements latéraux, canalisations des rivières et canaux créés entièrement, drainages, écluses et réservoirs, irrigations, consommation pour l'alimentation, l'hygiène, l'industrie, modifications des tapis végétaux par défrichement, déboisement, introduction de nouvelles cultures, etc..

Les Procédés (Hydrométrie et calculs par formules), grâce auxquels on détermine les débits ressortent de l'hydraulique générale appliquée. Ils méritent de prendre une place d'honneur dans les bons textes consacrés à la Potamologie générale ou appliqués à une seule rivière, ou à un ensemble fluvial bien délimité et plus ou moins homogène ou divers.

Et nous avons adopté l'habitude de distinguer dans l'Hydrologie fluviale générale trois parties : les Facteurs des régimes, les Mesures et calculs Hydrométriques et les Eléments des régimes qui sont à vrai dire les objets principaux, les fins de cette science.

(1) Le terme général de fleuves, pour toutes les eaux courantes, serait en accord avec le mot : fluvial, employé pour n'importe quel cours d'eau selon notre coutume. Mais si nous appelons sans trop de gêne : rivières, les plus infimes ruisseaux ou les fleuves les plus imposants, nous ne pouvons nous résoudre à qualifier de: fleuves des artères minuscules de drainage.

Tableau 7. Abondance - 2 - Les Bilans

\section{PREMIĖRE PARTIE}

\section{LES BILANS ANNUELS PRÉCIPITATIONS-DÉBITS}

Matières étudiées dans ces chapitres

\section{CHAPITREI - DÉFINITIONS ET QUELQUES} FAITS PRIMORDIAUX

A. Terminologie

Les indices, les quotients et les déficits d'écoulement

B. Différences entre l'évapotranspiration vraie et le déficit d'écoulement

1 - L'évapotranspiration, cause générale des différences entre précipitations et écoulements

2 - Déficit, et évapotranspiration vraie, exacte, intégrale ou contemporaine

3 - Les mises en réserve ou retenues, et les restitutions perturbatrices des bilans

C. Nature des retenues et origine des restitutions

1 - Accumulations souterraines de réserves hydrologiques, perméabilité, porosité, infiltrations

a) Le problème général de la perméabilité

b) Quelques chiffres-types de porosité brute

c) Vitesses d'infiltration et de percolation. Le coefficient de Darcy

d) Porosité nette, capillarité, nappes phréatiques ou captives

e) Perméabilité effective

f) Réserves hydrologiques et réserves agrico les, ou inutilisables pour les rivières

2 - Accumulations temporaires non souterraines. Lacs, neiges et glaces

a) Conséquences de ruissellements très ralentis

b) Mises en réserves lacustres

c) Accumulations nivales

d) Rétention glaciaire

D. Les équations-types des bilans précipitationsdébits

1 - Bilans de la mise en réserve et de la restitution

2 - L'équation vraie du bilan annuel particulier

3 - Identité approximative du déficit et de l'évapotranspiration pour le bilan global d'une longue suite d'années pages

2

2

5

6

6

8

11
CHAPITRE ॥- LES FACTEURS DU DÉFICIT ANNUEL D'ÉCOULEMENT PRECIPITATIONS, TEMPÉRATURES, HUMIDITÉ

Remarques préliminaires

A. L'abondance pluviale

B. Températures elles-mêmes influencées par l'altitude

1 - L'effet essentiel. Déficit variant dans le même sens que la température

2 - Exceptions insignifiantes dans l'ensemble à la relation générale susdite de cause à effet

C. Répartitions mensuelles des pluies

D. Effets combinés des températures et des précipitations totales et saisonnières

1 - Importance de ce facteur complexe

2 - Les indices d'aridité

3 - L'évapotranspiration potentielle. Ses diffé rences ou ses analogies avec l'évapotranspiration vraie

E. Autres quotients

1 - Fleuves et rivières de tous genres

2 - Le cas de l'Amazone

APPENDICE - INDICES D'ARIDITÉ ET D'HUMIDITÉ

1 - Définitions

2 - Formules de De Martonne

\begin{tabular}{ll|l|l} 
& ... & 142
\end{tabular}

3 - Qualités et insuffisances des formules de ${ }^{\cdots}$

a) Défauts et cependant valeur approximative déjà grande

b) Significations climatiques générales et phytogéographiques

c) Valeurs les plus fortes des indices en divers bassins

d) Assez bonnes relations possibles entre les indices en question et les modules spécifiques

e) Absence ou insuffisance des relations entre les indices et maintes espèces végé tales

4 - Diverses recherches et formules postérieures

a) Remarques générales

b) Formules d'Emberger et d'Angström $\quad$...

c) Formules basées sur l'évapotranspiration potentielle

5 - Formules de Gaussen

a) L'indice xérothermique

b) L'indice hygrothermique

Conclusion 
Tableau 8. Abondance - $5-$ Indices d'aridité $2^{\mathbf{e}}$ partie

\section{DEUXIĖME PARTIE}

\section{LES VALEURS DE L'ABONDANCE GLOBALE DANS LE MONDE}

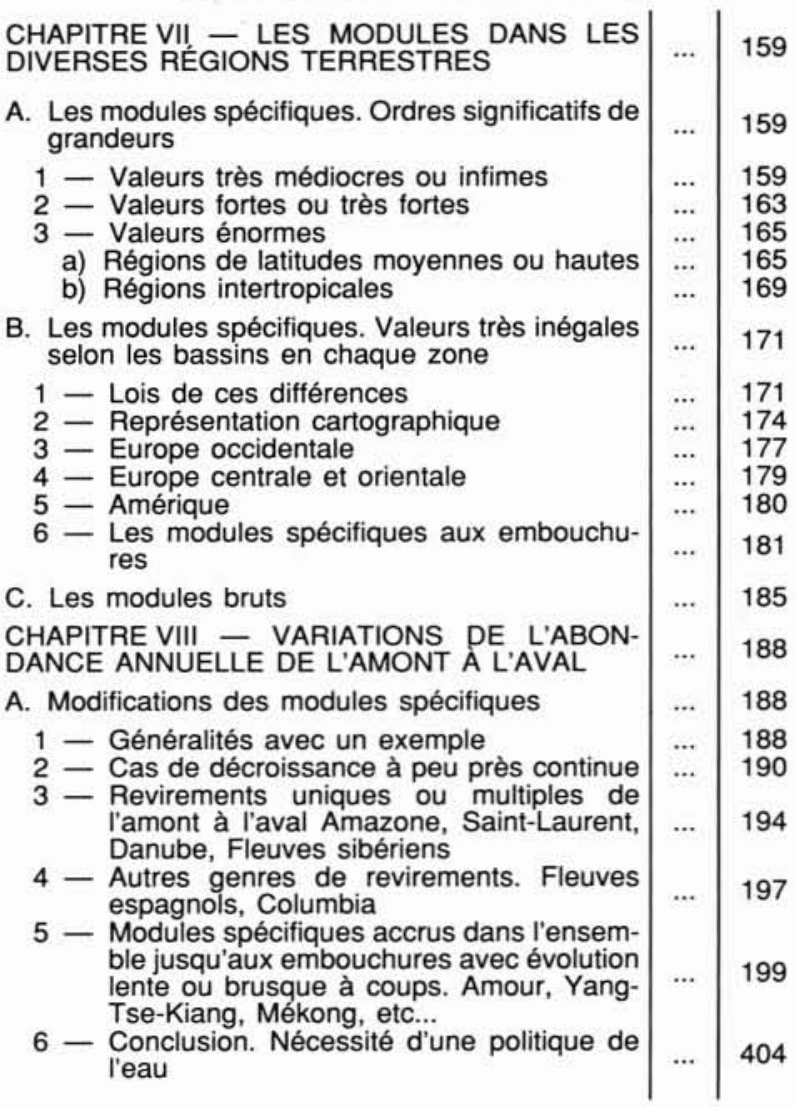

Tableau 9. Abondance $-11-4^{\circ}$ partie

\section{QUATRIĖME PARTIE}

\section{FORMULES ET MÉTHODE POURL'ÉVALUATION DES MODULES}

CHAPITRE XVII - FORMULES D'ÉVAPORATION

A. Evaporation et évapotranspiration sur les surfaces liquides

1 - Remarques générales

2 - Formules générales d'évaporation

3 - Moyens de mesure

a) Appareils divers

b) Les bacs

4 - Bilans observés sur les lacs

B. Formules d'évapotranspiration potentielle

1 - Remarques générales

2 - Formules de Thornthwaite

3 - Formules ultérieures dont celle de $L$. Turc CHAPITRE XVIII - FORMULES UTILISABLES POUR LES MODULES GLOBAUX

Remarques générales

A. Formules surtout théoriques

1 - Recours éventuellement utile aux formules d'évapotranspiration potentielle

a) Cas plus ou moins favorables à leur emploi

b) Quelques remarques sur les modes d'emploi

2 - Recours scabreux aux formules a priori d'évapotranspiration vraie

B. Formules essentiellement expérimentales

1 - Mode d'établissement

2 - Formules zonales ou régionales avec une seule variable

3 - Formules régionales avec deux variables

4 - Formules d'emploi général avec deux variables

C. Emploi des formules pour les bassins considérés à part

1 - Retouches nécessaires

2 - Bilans précipitations-débits transcendés grâce à des inductions fondées sur le relief

3 - Evaluations géographiques seulement relatives à certaines parties des bassins

D. Observations directes faites sur de petites surfaces et propres à guider les retouches par voie d'analogie

1 - Emploi des lysimètres

2 - Recours aux bassins naturels expérimentaux

CHAPITRE XIX - EXTENSIONS À LA NORMALE

A. Nécessité de contrôles et d'ajustements pour les modules globaux partiels

B. Possibilité fréquente des extensions à la normale

C. Calculs par les covariations

1 - Principes et exemples théoriques

2 - Cas de discordances

D. Calculs d'après les précipitations et les bilans vraisemblables

1 - Principes

2 - Modes de calcul
405

405

405

408

409 
Tableau 10. Abondance $-14-$ Conclusion

\section{CONCLUSION D'ENSEMBLE}

\begin{tabular}{|c|c|c|c|}
\hline MPLE & UR INSOLITE DE CE TEXTE CONCLUS & & 531 \\
\hline Eva & uation des modules particuliers & . & 533 \\
\hline $1-$ & Calcul par covariations & & 533 \\
\hline $2-$ & $\begin{array}{l}\text { Calcul par corrélations expérimentales } \\
\text { précipitations-débits }\end{array}$ & $\cdots$ & \\
\hline $3-$ & Choix d'une année hydrologique adéquate & $\cdots$ & 35 \\
\hline & $\begin{array}{l}\text { Calculs sans bases expérimentales sur les } \\
\text { débits locaux }\end{array}$ & $\cdots$ & 537 \\
\hline $\begin{array}{l}5- \\
6-\end{array}$ & $\begin{array}{l}\text { Contrôles et rectifications nécessaires } \\
\text { Cours d'eau glaciaires }\end{array}$ & $\cdots$ & $\begin{array}{l}53 \\
54\end{array}$ \\
\hline Evalt & uation des modules globaux normaux & ... & 4 \\
\hline $1-$ & $\begin{array}{l}\text { bases expérimentales loca- } \\
\text { ules géographiques }\end{array}$ & * & \\
\hline $2-$ & Inexactitudes p & $\ldots$ & \\
\hline $3-$ & Emp & & \\
\hline $4-$ & Cours d'eau glaciaires & & \\
\hline $5-$ & $\begin{array}{l}\text { Evaluations sans recours directs aux } \\
\text { facteurs climatiques }\end{array}$ & ... & \\
\hline $6-$ & $\begin{array}{l}\text { Extensions à la normale. Définitions et } \\
\text { réserves }\end{array}$ & ... & \\
\hline Varia & abilité des modules & ... & \\
\hline $1-$ & Rem & $\ldots$ & \\
\hline $2-$ & Fréquences et distributions & & \\
\hline & Coefficients moyens ou types de variation & $\cdots$ & \\
\hline $4-$ & $\begin{array}{l}\text { Rapports des modules particuliers } \\
\text { extrêmes }\end{array}$ & & \\
\hline $\begin{array}{l}5- \\
6-\end{array}$ & $\begin{array}{l}\text { Anomalies des modules globaux partiels } \\
\text { Très faible vraisemblance de périodicités } \\
\text { régulières }\end{array}$ & $\ldots$ & \\
\hline
\end{tabular}

\begin{tabular}{ll|l|l}
7 - Variabilité de l'abondance moyenne au & & \\
cours des évolutions terrestres & $\ldots 62$
\end{tabular}

a) Signification douteuse de certains symptômes

b) Stabilité approximative vraisemblable durant l'époque historique récente

c) Certitudes de grands changements pour les modules dans le passé et dans l'avenir

Affaiblissements imminents pour des causes artificielles

D. Les expressions numériques des bilans précipitations-débits dans le monde

1 - Les déficits globaux d'écoulement $\quad \ldots .565$

2 - Les déficits particuliers $\quad \ldots .568$

3 - Quotients d'écoulement $\quad \ldots . \mid 568$

\begin{tabular}{l|l|l} 
E. Les modules globaux dans le monde & $\ldots$ & 569
\end{tabular}

1 - Modules spécifiques $\quad \ldots .569$

a) Valeurs-types diverses $\quad$...

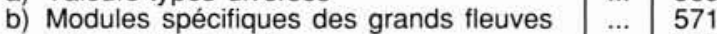

2 - Les modules bruts $\quad \ldots . \mid 572$

\begin{tabular}{l|l|l}
2 - Les modules bruts & $\ldots$ & 572 \\
3 - Apports totaux aux Océans & $\ldots$ & 573
\end{tabular}

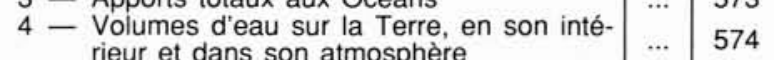

\begin{tabular}{ll|l} 
Remarques finales. Recherches les plus importan- & & \\
.. & 575
\end{tabular}

\begin{tabular}{l|l|l} 
tes à faire sur l'abondance et sur les bilans & $\ldots$ & 575 \\
BIBLIOGRAPHIE SOMMAIRE & $\ldots$ & 576
\end{tabular} 